\title{
Introduction to the special issue on search as learning
}

\author{
Carsten Eickhoff ${ }^{1} \cdot$ Jacek Gwizdka $^{2}$ - Claudia Hauff ${ }^{3}$ • \\ Jiyin $\mathbf{H e}^{4}$
}

Received: 29 July 2017 / Accepted: 23 August 2017/Published online: 9 September 2017

(C) Springer Science+Business Media, LLC 2017

The Internet connects vast knowledge resources that are assumed to contain the necessary information to answer most general questions (Kräenbring et al. 2014; Reavley et al. 2012). However, even given this seemingly inexhaustible well of information, the act of learning from it consists of more than just looking-up and memorizing facts. Constructivist theoreticians including Piaget and Vygotsky argue that the learning process necessarily depends on the context of existing knowledge upon which the newly encountered factoids are built. The stronger the contextualization of new knowledge, the more effortless and effective learning is assumed to be. Jean Piaget first proposed the theory of "Cognitive Development" that considers knowledge to be an actively constructed complex system of experience, stage of cognitive development, cultural background and personal history (Piaget 1976). In other words, knowledge is derived from personal experience and ideas rather than an aggregation of loose facts and formulas. Building on Piaget's theories, Johnson (2002) and Langley (1995) study order effects in incremental learning. The authors find that the order in which material is consumed has a significant influence on the overall learning rate and absolute knowledge retention. Kuhlthau (1993) discusses the importance of mediators who enable learners to go beyond the current limits of their understanding. Despite the wide acceptance and demonstrated success of constructivist methods in pedagogics, common retrieval models do not explicitly manifest any notion of contextual learning. Document relevance is largely judged in isolation and list-wide ranking considerations rarely go beyond diversification efforts. Consequently, state-of-the-

Carsten Eickhoff

c.eickhoff@acm.org

1 ETH Zurich, Zurich, Switzerland

2 University of Texas at Austin, Austin, TX, USA

3 Delft University of Technology, Delft, The Netherlands

4 Centrum Wiskunde and Informatica (CWI), Amsterdam, The Netherlands 
art search engines cannot be considered ideal learning environments. Over the past decade, the study of users' domain-specific knowledge has led to a wide number of innovations in user behavior understanding. White et al. (2009) show that, within their area of expertise, domain experts search differently from non-experts. They are found to use a more diverse vocabulary of query terms and generally demonstrate a better understanding of the desired results to be retrieved, resulting in improved query formulation and result inspection performance as compared to laypeople. Such domain expertise is by no means a static notion and a number of studies have noted considerable long-term changes (Liu et al. 2013; Wildemuth 2004) as well as more gradual within-session developments (Eickhoff et al. 2009, 2015). This is assumed to occur as a consequence of repeatedly being exposed to in-domain information, e.g., during Web search, and therefore having acquired increased domain expertise. While these works laid foundations towards knowledge acquisition in search, the dedicated academic interest in construing searching as a learning process, in recognizing the importance of learning as a search outcome and in providing explicit support for learning while searching is a rather recent development. The initial ideas were presented at recent workshops (Freund et al. 2013; Freund et al. 2014; Gwizdka et al. 2016), in a special issue of Journal of Information Science (Hansen and Rieh 2016) as well as during a Dagstuhl Seminar. ${ }^{1}$ This special issue continues these themes and brings them to the information retrieval community by presenting six novel research efforts into expertise-based search strategies and support, collaborative learning paradigms and retrieval technology that bridges the effectiveness gap between expert and novice searchers.

\section{Accepted articles}

Kodama et al. (2017) investigate the relationship between middle school students' mental models of Google and their information seeking skills. Students tended to personify Google and showed a limited understanding of how search engines work. This research suggests to developers and interface designers to make the inner workings of the engine more transparent and has implications on digital literacy instruction. Lu and Hsiao (2017) study the information seeking behavior of users in programming language forums. The authors also devise a personalized information seeking assistant that fosters learning through user behavior modeling and query refinement, showing significantly improved learning effectiveness. Karanam et al. (2017) present a search result click prediction model incorporating the user's individual level of relevant domain knowledge. The authors further discuss and compare strategies for knowledge acquisition tailored to the existing state of knowledge, showing significant gains in knowledge acquisition when using expertiseadequate strategies.

Knight et al. (2017) investigate the usefulness of orchestration, a framework explicitly manifesting background context, actors and activities, for collaborative learning and searching. The authors' empirical evaluation is set in a classroom environment and promises beneficial outcomes for use in educational institutions.

Syed and Collins-Thompson (2017) study retrieval methods that are optimized for enhancing the user's learning efficiency by diversifying relevant search results in terms of vocabulary usage. A crowdsourced user study demonstrates significant learning gains when rankers include a high density of previously unfamiliar new terms. Pera et al. (2017) present an enhanced search environment YouUnderstood.Me (YUM) that aims to support

\footnotetext{
${ }^{1}$ http://www.dagstuhl.de/17092.
} 
learning of K-9 children through retrieving documents that not only match children information needs but also their reading abilities. The article takes a user-centered approach and makes a distinction between searching to learn and learning to search.

\section{Conclusion}

The six contributions to this special issue span a wide range of domain-specific studies, demonstrating a significant potential for studying search as an information seeking and learning activity. Each of them makes a strong case for modeling learning intents and activities in the context of information search and retrieval. In the future, further empowering learning efforts by means of technology will critically depend on our ability as a community to recognize and adequately represent instances of knowledge acquisition as they occur in real-life information systems. Especially the study of user models and personalization, not just on a topical, but also a domain-expertise level will be a key factor in this regard.

\section{References}

Azpiazu, I. M., Dragovic, N., Pera, M. S., \& Fails, J. A. (2017). Online searching and learning: YUM and other search tools for children and teachers. Information Retrieval. doi:10.1007/s10791-017-9310-1.

Eickhoff, C., Dungs, S., \& Tran, V. (2015). An eye-tracking study of query reformulation. In Proceedings of the 38th international ACM SIGIR conference on research and development in information retrieval, ACM, pp. 13-22.

Eickhoff, C., Teevan, J., White, R., \& Dumais, S. (2014). Lessons from the journey: A query log analysis of within-session learning. In Proceedings of the 7th ACM international conference on web search and data mining, ACM, pp. 223-232.

Freund, L., Gwizdka, J., Hansen, P., Kando, N., \& Rieh, S. Y. (2013). From searching to learning. In M. Agosti, N. Fuhr, E. Toms, \& P. Vakkari (Eds.), Evaluation methodologies in information retrieval. Dagstuhl reports (Vol. 13441, pp. 102-105). http://drops.dagstuhl.de/opus/volltexte/2014/4433.

Freund, L., He, J., Gwizdka, J., Kando, N., Hansen, P., \& Rieh, S. Y. (2014). Searching as learning (SAL) workshop. In Proceedings of the 5th information interaction in context symposium IIiX '14, ACM, pp. 7-7.

Gwizdka, J., Hansen, P., Hauff, C., He, J., \& Kando, N. (2016). Search as learning (SAL) workshop . In Proceedings of the 39th international ACM SIGIR conference on research and development in information retrieval, SIGIR '16, ACM, pp. 1249-1250.

Hansen, P. \& Rieh, S. Y. (2016). Recent advances on searching as learning: An introduction to the special issue. London, UK: Sage Publications.

Johnson, E. B. (2002). Contextual teaching and learning: What it is and why it's here to stay. Thousand Oaks: Corwin Press.

Karanam, S., Jorge-Botana, G., Olmos, R., \& van Oostendorp, H. (2017). The role of domain knowledge in cognitive modelingof information search. Information Retrieval. doi:10.1007/s10791-017-9308-8.

Knight, S., Rienties, B., Littleton, K., Tempelaar, D., Mitsui, M., \& Shah, C. (2017). The orchestration of a collaborative information seeking learning task. Information Retrieval. doi:10.1007/s10791-017-9304$\mathrm{z}$.

Kodama, C., St. Jean, B., Subramaniam, M., \& Taylor, N. G. (2017). There's a creepy guy on the other end at Google!: Engaging middle school students in a drawing activity to elicit their mental models of Google. Information Retrieval. doi:10.1007/s10791-017-9306-x.

Kräenbring, J., Penza, T. M., Gutmann, J., Muehlich, S., Zolk, O., Wojnowski, L., et al. (2014). Accuracy and completeness of drug information in wikipedia: A comparison with standard textbooks of pharmacology. PLoS ONE, 9(9), e106930.

Kuhlthau, C. C. (1993). Seeking meaning. Norwood, NJ: Ablex.

Langley, P. (1995). Order effects in incremental learning. Learning in Humans and Machines: Towards an Interdisciplinary Learning Science, Pergamon, 136, 137. 
Liu, J., Belkin, N. J., Zhang, X., \& Yuan, X. (2013). Examining users' knowledge change in the task completion process. Information Processing \& Management, 49(5), 1058-1074.

Lu, Y. \& Hsiao, I. H. (2017). Personalized information seeking assistant (PiSA): From programming information seeking to learning. Information Retrieval. doi:10.1007/s10791-017-9305-y.

Piaget, J. (1976). Piaget's theory. Berlin: Springer.

Reavley, N. J., Mackinnon, A. J., Morgan, A. J., Alvarez-Jimenez, M., Hetrick, S. E., Killackey, E., et al. (2012). Quality of information sources about mental disorders: A comparison of wikipedia with centrally controlled web and printed sources. Psychological Medicine, 42(08), 1753-1762.

Syed, R. \& Collins-Thompson, K. (2017). Optimizing search results for human learning goals. Information Retrieval. doi:10.1007/s10791-017-9303-0.

White, R. W., Dumais, S. T, \& Teevan, J. (2009). Characterizing the influence of domain expertise on web search behavior. In Proceedings of the second ACM international conference on web search and data mining, ACM, pp. 132-141.

Wildemuth, B. M. (2004). The effects of domain knowledge on search tactic formulation. Journal of the American Society for Information Science and Technology, 55(3), 246-258. 